\title{
Controle de Murdannia nudiflora em pós colheita da soja ${ }^{1}$
}

\section{Murdannia nudiflora control in soybean postharvest}

\author{
Marcelo Raphael Volf ${ }^{2}$; Fellipe Goulart Machado ${ }^{3}$; Rafael Locatelli ${ }^{4}$; Vinicius Marca Marcelino \\ de Lima ${ }^{5}$; Eliezer Antonio Gheno ${ }^{3}$; Rafael Romero Mendes ${ }^{6}$; Sergio de Oliveira Procópio ${ }^{7}$
}

Resumo - A espécie Murdannia nudiflora pertence à família Commelinaceae é conhecida como "trapoerabinha". Devido à elevada capacidade competitiva e por apresentar tolerância ao glyphosate esta espécie se desenvolve e compete com a soja, apresenta rápido crescimento quando ocorre a senescência das folhas de soja e dificulta o manejo das plantas daninhas no cultivo subsequentes. O objetivo deste trabalho foi avaliar a eficiência de herbicidas isolados ou em associação em pós colheita da soja e verificar a dose do ingrediente ativo que proporciona o melhor controle de $M$. nudiflora. Foram realizados dois experimentos a campo, em delineamento de blocos casualizados com quatro repetições. O primeiro experimento foi constituído por oito tratamentos sendo eles glyphosate $972 \mathrm{~g}$ e.a. ha ${ }^{-1}$, atrazine $1500 \mathrm{~g}$ i.a. ha ${ }^{-1}$; carfentrazone $12 \mathrm{~g}$ i.a. ha- ${ }^{-1}$, imazethapyr $100 \mathrm{~g}$ i.a. ha ${ }^{-1}$, glyphosate + imazethapyr $972+30 \mathrm{~g} \mathrm{ha}^{-1}$, imazethapyr + atrazine 30 $+1500 \mathrm{~g} \mathrm{ha}^{-1}$, carfentrazone + atrazine $8+1500 \mathrm{~g} \mathrm{ha}^{-1}$ e testemunha. No segundo experimento, utilizou-se atrazine nas doses de $0,880,1.100,1.320$ e 1.540 g i.a. ha ${ }^{-1}$. Em todos os experimentos as aplicações dos herbicidas foram realizadas em pós-emergência das plantas daninhas. Nos resultados do primeiro experimento, apenas os tratamentos com atrazine apresentaram controle eficiente (> 80\%). O glyphosate na dose de $972 \mathrm{~g}$ e.a. ha ${ }^{-1}$ apresentou baixo controle $(<20 \%)$ mesmo quando em mistura com imazethapyr. No segundo experimento, doses a partir de $880 \mathrm{~g}$ i.a. ha $^{-1}$ de atrazine foram suficientes para o controle de $M$. nudiflora em pós-emergência.

Palavras-chaves: atrazine; Commelinaceae; glyphosate

Abstract - The species of Murdannia nudiflora belongs to the family Commelinaceae is known as doveweed. Because of competitive capacity and tolerance to glyphosate this species growing during the soybean crop cycle, presenting rapid growth when the leaf senescence of soybean occurs difficulting weed management in others cultures. The objective of this study was to evaluate the efficiency of different active ingredients of herbicides in combination or separately, and verify the best dosage of the active ingredient, which provided the best control of M. nudiflora. Two field experiments were arranged in randomized block design. The first experiment consisted of eight herbicide treatments being glyphosate $972 \mathrm{~g}$ a.e. $\mathrm{ha}^{-1}$, atrazine 1500 a.i. $\mathrm{g} \mathrm{ha}^{-1}$, carfentrazone $12 \mathrm{~g}$

\footnotetext{
${ }^{1}$ Recebido para publicação em 25/10/2016 e aceito em 05/12/2016.

${ }^{2}$ Doutorando pela UNESP - FCA - Botucatu SP. E-mail: <marcelovolf@ gmail.com>.

${ }^{3}$ Doutorando em Agronomia. Núcleo de Estudos Avançados em Ciência das Plantas Daninhas (NAPD/UEM), Maringá, PR, Brasil. E-mail: <fellipe.goulart@outlook.com; eliezer.gheno@ gmail.com>.

${ }^{4}$ Engenheiro Agrônomo pela UNEMAT - Nova Xavantina - MT. E-mail: <lacatellinx@ gmail.com>.

5 Me. Eng. Agrônomo das Faculdades Unidas do Vale do Araguaia, Barra do Garças - MT. E-mail: <vinimarca@gmail.com>.

${ }^{6}$ Mestrando em Agronomia. Núcleo de Estudos Avançados em Ciência das Plantas Daninhas (NAPD/UEM), Maringá, PR, Brasil. E-mail: <rafaromero.mendes@gmail.com>.

${ }^{7}$ Prof. Dr. Eng. Agrônomo da Universidade de Rio Verde, Rio Verde - GO. E-mail: <procopio.so@gmail.com>.
} 
a.i. ha ${ }^{-1}$, imazethapyr $100 \mathrm{~g}$ a.i. ha ${ }^{-1}$, glyphosate + imazethapyr $972+30 \mathrm{~g} \mathrm{ha}^{-1}$, imazethapyr + atrazine $30+1500 \mathrm{~g} \mathrm{ha}^{-1}$, carfentrazone + atrazine $8+1500 \mathrm{~g} \mathrm{ha}^{-1}$ and check. The second experiment used the herbicide atrazine in different dosages $0,880,1100,1320$ e $1540 \mathrm{~g}$ a.i. ha ${ }^{-1}$. In the experiments all herbicide was applied in post emergence. In the results of the first experiment, only treatments with atrazine presented efficient control (> 80\%). Glyphosate at the dose of $972 \mathrm{~g}$ a.e. $\mathrm{ha}^{-1}$ showed low control $(<20 \%)$ even when mixed with imazethapyr. In the second experiment, doses starting $880 \mathrm{~g}$ a.i. ha ${ }^{-1}$ of atrazine were sufficient for control in postemergence of $M$. nudiflora.

Keywords: atrazine; Commelinaceae; glyphosate

\section{Introdução}

A família Commelinaceae abrange de 40 a 50 gêneros, com aproximadamente 500 espécies. $\mathrm{Na}$ agricultura brasileira quatro espécies desta família apresentam importância agronômica, dentre elas a espécie Murdannia nudiflora (L.) Brenan, tem aumentado a infestação no estado do Mato Grosso, gerando interesse, sobre o manejo desta planta daninha (Lorenzi, 2008).

A $M$. nudiflora é conhecida popularmente como trapoerabinha ou alho bravo. Apresenta hábito de crescimento anual e se reproduz por sementes e órgãos vegetativos (estolões). O desenvolvimento vegetativo é lento até os 30 dias e depois disso, ocorre a formação de estolões e seu desenvolvimento passa a ser mais vigoroso. Ao longo do seu ciclo uma planta pode produzir em aproximadamente 4.600 sementes (Kissmann e Groth, 1997; Erasmo et al., 2003; Lorenzi, 2008).

Espécies de plantas daninhas pertencentes à família Commelinaceae, apresentam alto potencial de interferência, na cultura da soja, densidades de 4 plantas $\mathrm{m}^{2}$ de Commelina benghalensis podem reduzir a produtividade em até 25,7 \% (Osipe et al., 2008). Para o milho, a mesma espécie, apresenta interferência na absorção de nutrientes reduzindo o conteúdo de macro e micronutrientes nos tecidos foliares (Cury et al., 2012).

Nos Estados Unidos, mais precisamente na região da Carolina do Norte, vem ocorrendo aumento da infestação da espécie, principalmente em grandes culturas como algodão, soja e milho. Isso ocorre devido aos sistemas de produção utilizados, como semeadura convencional, os quais esta espécie é adaptada e também devido a tolerância ao herbicida glyphosate (Wilson Jr. et al., 2006). No Brasil foram relatadas a presença desta planta daninha em lavouras de soja e arroz de sequeiro no Mato Grosso (Ferraresi et al., 2009; Luz et al., 2014), arroz irrigado no sul do Tocantins (Erasmo et al., 2008) e Roraima (Cruz et al., 2009). O aumento na infestação de $M$. nudiflora deve-se principalmente as características preferenciais inerentes a ela, tais como tipo de reprodução e rápido desenvolvimento vegetativo (Kismman e Groth, 1997). Outro fator que favorece ao aumento na infestação de $M$. nudiflora em sistemas agrícolas é a utilização de culturas geneticamente modificadas, tolerantes ao glyphosate, onde o manejo de espécies invasoras é realizado basicamente com a aplicação de glyphosate. O glyphosate quando aplicado em plantas de $M$. nudiflora em estádios de desenvolvimento inicial (3 a 7 folhas) apresenta controle insatisfatório, evidenciando a elevada tolerância ao herbicida e a necessidade da utilização de outros métodos de controle (Ahmed et al., 2015).

$\mathrm{O}$ aumento da infestação de $M$. nudiflora em sistemas de plantio direto na região do Mato Grosso, acontece principalmente pela não adoção de estratégias de manejo como rotação de culturas e plantio direto, o que facilita a adaptação da espécie ao sistema utilizado (Lorenzi, 2008). A integração de manejos culturais, como por exemplo, aumento da palhada no solo e uso de herbicidas de alta 
eficiência de controle, se tornam necessários para redução da infestação por $M$. nudiflora (Luz et al., 2014). Porém deve-se atentar ao uso dos herbicidas, preservando a eficiência de controle pela rotação de mecanismos de ação e prevenção da seleção de biótipos resistentes (Norsworthy et al., 2012).

No Brasil, apenas três herbicidas são registrados para o controle de $M$. nudiflora: glyphosate, bentazon e atrazine (MAPA, 2016a). A pouca quantidade de herbicidas registrados para o controle de $M$. nudiflora, aliada ao aumento da infestação da espécie no Brasil instiga a pesquisa a buscar novas opções para o manejo desta planta daninha (Erasmo et al., 2003). Além disso, não existem informações sobre a resposta de controle frente a aplicação de herbicidas em associação. Destaca-se também, que apesar dos herbicidas glyphosate, atrazine e bentazon, serem registrados para o controle, existe pouca informação disponível sobre a eficácia agronômica destes produtos.

Visto as problemáticas existentes em relação a $M$. nudiflora e a escassez de informação sobre a espécie nos sistemas de cultivo do Brasil, este trabalho teve como objetivo avaliar a eficiência de herbicidas isolados ou em associação em pós colheita da cultura da soja, e verificar a dose do herbicida que proporciona o melhor controle de $M$. nudiflora.

\section{Material e Métodos}

Foram conduzidos dois experimentos a campo no município de Nova Xavantina (MT), com coordenadas $14^{\circ} 45^{\prime} \mathrm{S}, 52^{\circ} 21^{\prime} \mathrm{O}$, e altitude de 320 metros. O clima é Aw, segundo a classificação de Köppen, se caracterizando por ser temperado úmido com inverno seco e verão quente. Os dados meteorológicos coletados durante a condução do experimento estão dispostos na Figura 1.

O primeiro experimento foi conduzido durante os meses de abril a maio de 2009, visando avaliar o controle de $M$. nudiflora com diferentes tratamentos herbicidas em manejo pós-colheita da cultura da soja. O delineamento utilizado foi de blocos casualizados (DBC), com quatro repetições e oito tratamentos (Tabela 1). As unidades experimentais continham $25 \mathrm{~m}^{2}$ de área total $(5 \times 5 \mathrm{~m})$, sendo a área útil para as avaliações de $20 \mathrm{~m}^{2}$, área onde foi realizada a aplicação dos tratamentos.
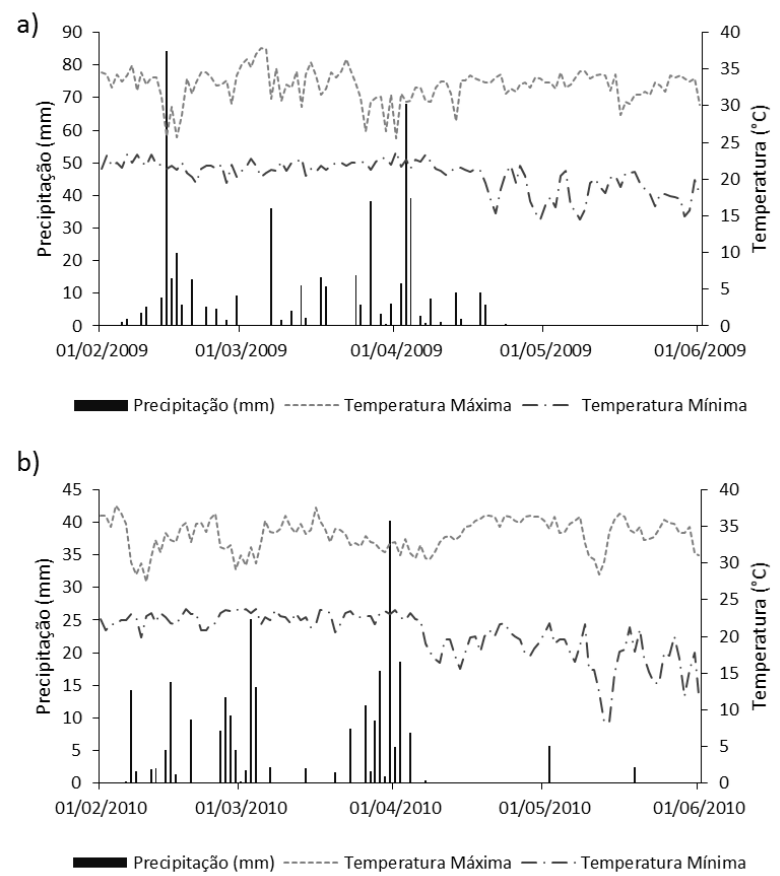

Figura 1. Regime pluviométrico e temperaturas durante o período de condução do experimento na safra 2009 (a) (Experimento 1) e em 2010 (b) (Experimento 2), Nova Xavantina (MT).

O segundo experimento foi instalado na mesma propriedade do experimento 1 (mesmo talhão) onde foi constatada a maior infestação da planta daninha alvo, sendo conduzido entre os meses de março a maio do ano de 2010. O experimento foi instalado no delineamento de blocos casualizados, com quatro repetições e constituído de cinco tratamentos, sendo: 1. Testemunha sem aplicação de herbicida; 2 . atrazine $\left(880 \mathrm{~g}\right.$ i.a. $\left.\mathrm{ha}^{-1}\right)$; 3. atrazine $(1.100 \mathrm{~g}$ i.a. $\left.\mathrm{ha}^{-1}\right)$; 4. atrazine $\left(1.320 \mathrm{~g}\right.$ i.a. ha $\left.{ }^{-1}\right)$; e 5. atrazine (1.540 g i.a. ha $\left.{ }^{-1}\right)$. O produto comercial utilizado nos tratamentos foi Gesaprim, 880 g i.a. $\mathrm{L}^{-1}$, GRDA, Syngenta. As dimensões das parcelas e 
a alocação da área útil foram as mesmas às descritas para o experimento 1 .

Nos dois experimentos as aplicações dos herbicidas foram realizadas a duas semanas após a colheita da soja, quando as plantas de $M$. nudiflora estavam em pleno florescimento.
Estas se encontravam em estádio de desenvolvimento avançado devido a capacidade de se desenvolverem durante o ciclo da cultura da soja, principalmente após a senescência das folhas da cultura, onde há o aumento da incidência de luz solar.

Tabela 1. Relação de tratamentos avaliados no experimento de controle químico de Murdannia nudiflora. Experimento 1. Nova Xavantina (MT), 2009.

\begin{tabular}{|c|c|c|}
\hline Tratamentos & Dose g i.a. ou e.a. ha ${ }^{-1}$ & Produto comercial (Fabricante) \\
\hline 1. Glyphosate ${ }^{1 /}$ & 972 & Roundup Ready CS (Monsanto) \\
\hline 2. Atrazine ${ }^{1 /}$ & 1500 & Atrazina Nortox 500 SC (Nortox) \\
\hline 3. Carfentrazone ${ }^{1 /}$ & 12 & Aurora CE (FMC) \\
\hline 4. Imazethapyr ${ }^{1 /}$ & 100 & Pivot CS (Basf) \\
\hline 5. Glyphosate + imazethapyr ${ }^{1 /}$ & $972+30$ & Roundup Ready CS (Monsanto) + Pivot CS (Basf) \\
\hline 6. Imazethapyr + atrazine $e^{1 /}$ & $30+1500$ & Pivot CS (Basf) + Atrazina Nortox 500 SC (Nortox) \\
\hline 7. Carfentrazone + atrazine $e^{1 /}$ & $8+1500$ & Aurora CE $(\mathrm{FMC})+$ Atrazina Nortox $500 \mathrm{SC}$ (Nortox) \\
\hline 8. Testemunha s/ herbicida & - & - \\
\hline
\end{tabular}

Para as pulverizações se utilizou pulverizador costal de pressão constante à base de $\mathrm{CO}_{2}$, equipado com barra contendo quatro pontas de pulverização do tipo jato plano simples XR-110.02 espaçadas entre si de $0,5 \mathrm{~m}$ (faixa de aplicação de $2 \mathrm{~m}$ ), sob pressão de 394 $\mathrm{kPa}$. Estas condições de aplicação proporcionaram o equivalente a $200 \mathrm{~L} \mathrm{ha}^{-1} \mathrm{de}$ volume de calda.

No experimento 1 a aplicação de pósemergência para o controle da planta daninha foi realizada no dia 04/04/2009, as 10:00 h, umidade de $75 \%$, temperatura $28,2^{\circ} \mathrm{C}$ e vento a $3,4 \mathrm{~km} \mathrm{~h}^{-1}$. No experimento 2, a aplicação foi realizada no dia 23/03/2010, as 9:30 h, umidade de $78 \%$, temperatura $27,8^{\circ} \mathrm{C}$ e vento a $1,8 \mathrm{~km} \mathrm{~h}^{-}$ 1 .

Nos dois experimentos foram realizadas avaliações do controle de $M$. nudiflora aos sete, 14 e 28 dias após a aplicação (DAA), conforme metodologia proposta pela SBCPD (1995), por meio de escala visual de $0-100 \%$, onde, $0 \%$ significa ausência de sintomas e $100 \%$ morte de todas as plantas daninhas presentes na área útil da parcela. Para o controle ser considerado eficiente, o mesmo deve ser superior a $80 \%$.

No experimento 2, foi avaliado o percentual de cobertura do solo pela planta daninha, antes da aplicação dos tratamentos, com auxílio de um quadrado vazado de 1 x $1 \mathrm{~m}$ subdividido em 100 quadrículos de $100 \mathrm{~cm}^{2}$. O quadro foi lançado duas vezes ao acaso dentro de cada parcela e contado quantos quadrículos estavam preenchidos com a planta daninha, e assim foi gerada uma média percentual de cobertura em cada parcela. Foi avaliado a massa seca da parte aérea de $M$. nudiflora antes da aplicação para verificar a distribuição destas plantas nas parcelas experimentais, para isso se utilizou um quadrado vazado de $0,3 \times 0,3 \mathrm{~m}$, lançado duas vezes dentro da área útil de cada parcela, sendo coletada toda fitomassa da parte aérea da planta daninha presente dentro dessa área. Após a coleta o material vegetal foi acondicionado em estufa de circulação forçada a $65{ }^{\circ} \mathrm{C}$, e mantido até atingir peso constante. Após a retirada do material, este foi imediatamente pesado em balança de precisão.

Após a coleta e tabulação dos dados, os dados dos experimentos foram submetidos ao teste de normalidade. Os dados das avaliações realizadas apresentaram distribuição normal, assim procedeu-se a análise de variância e teste $\mathrm{F}$ com a probabilidade de $5 \%$. No primeiro experimento os tratamentos foram comparados ao teste de Tukey a 5\% de probabilidade, e no 
segundo experimentou procedeu-se a análise de regressão a 5\% de probabilidade. As análises estatísticas foram realizadas com auxílio do programa Genes (Cruz, 2016).

\section{Resultados e Discussão}

\section{Experimento 1. Eficácia dos herbicidas}

No primeiro experimento foi observado, em todas as épocas de avaliação, diferenças significativas para controle de $M$. nudiflora em função dos herbicidas aplicados isoladamente ou em associação (Tabela 2). Aos sete DAA, verificou-se que as associações de atrazine (1.500 g i.a. ha $\left.{ }^{-1}\right)$ com imazethapyr (30 g i.a. ha ${ }^{-}$ 1) ou carfentrazone $\left(8 \mathrm{~g}\right.$ i.a. $\left.\mathrm{ha}^{-1}\right)$ proporcionaram os melhores resultados de controle (até $70 \%$ ), todavia não diferindo do tratamento que continha somente atrazine (1500 gi.a. ha $\left.{ }^{-1}\right)$.

Nas avaliações posteriores (14 e 28 DAA) os tratamentos com atrazine, proporcionaram efeito progressivo de controle, resultando em morte total das plantas aos 28 DAA. Para os demais tratamentos foi observado menores percentuais de controle.

Tabela 2. Avaliação visual de controle (\%) de Murdannia nudiflora após a utilização de diferentes herbicidas. Experimento 1. Nova Xavantina (MT), 2009.

\begin{tabular}{|c|c|c|c|c|}
\hline \multirow{2}{*}{ Tratamentos } & \multirow{2}{*}{ Dose g i.a. ou e.a. ha- ${ }^{-1}$} & \multicolumn{3}{|c|}{$\%$ de controle - DAA* } \\
\hline & & 7 & 14 & 28 \\
\hline 1. Glyphosate & 972 & $20 \mathrm{~cd}$ & $20 \mathrm{bc}$ & $20 \mathrm{~b}$ \\
\hline 2. Atrazine & 1500 & $60 \mathrm{ab}$ & $95 \mathrm{a}$ & $100 \mathrm{a}$ \\
\hline 3. Carfentrazone & 12 & $20 \mathrm{~cd}$ & $5 \mathrm{c}$ & $5 \mathrm{c}$ \\
\hline 4. Imazethapyr & 100 & $20 \mathrm{~cd}$ & $5 \mathrm{c}$ & $5 \mathrm{c}$ \\
\hline 5. Glyphosate + imazethapyr & $972+30$ & $40 \mathrm{bc}$ & $30 \mathrm{~b}$ & $0 \mathrm{~d}$ \\
\hline 6. Imazethapyr + atrazine & $30+1500$ & $70 \mathrm{a}$ & $95 \mathrm{a}$ & $100 \mathrm{a}$ \\
\hline 7. Carfentrazone + atrazine & $8+1500$ & $70 \mathrm{a}$ & $95 \mathrm{a}$ & $100 \mathrm{a}$ \\
\hline 8. Testemunha sem herbicida & - & $0 \mathrm{~d}$ & $0 \mathrm{c}$ & $0 \mathrm{c}$ \\
\hline $\mathrm{CV}(\%)$ & & 26,6 & 21,8 & 15,7 \\
\hline
\end{tabular}

Médias seguidas pelas mesmas letras na coluna não diferem estatisticamente entre si, pelo teste de Tukey a $5 \%$ de probabilidade.

A tendência de maior controle das plantas de $M$. nudiflora observado aos 7 DAA, com a utilização de carfentrazone ou imazethapyr associados a atrazine, não se manteve nas avaliações posteriores (Tabela 2), indicando que a aplicação isolada de atrazine é suficiente para proporcionar excelente controle de $100 \%$. Diante desse fato, destaca-se a importância de se inserir culturas tolerantes a atrazine nos sistemas de produção de grãos em áreas infestadas com $M$. nudiflora, como milho e sorgo.

A aplicação de glyphosate não diferiu da testemunha aos 7 e 14 DAA, apresentando baixos níveis de controle $(<20 \%)$. Um fator determinante para o controle de plantas daninhas é o estádio no qual elas se encontram no momento da aplicação (Braz et al., 2012). A ineficiência do glyphosate pode ser devido ao estádio de desenvolvimento das plantas de $M$. nudiflora que estavam em pleno florescimento quando recebeu a aplicação dos tratamentos e também a dose utilizada. Em Commelina benghalensis o estádio de desenvolvimento tem efeito direto na tolerância da planta ao glyphosate (Dias et al., 2013). Quanto maior for o estádio de desenvolvimento de $C$. benghalensis, menor é a absorção e translocação do herbicida, podendo haver também metabolismo interno do glyphosate a outros compostos como AMPA (ácido aminometilfosfônico), reduzindo a toxicidade do herbicida (Monquero et al., 2004; Dias et al., 2013). Na literatura existem relatos demonstrando a baixa eficácia no controle de $M$. nudiflora em resposta ao herbicida glyphosate em doses de até $2.000 \mathrm{~g}$ e.a. ha ${ }^{-1}$ (Chauhan e Abugho, 2013; Ahmed et al., 2015). Tais 
resultados poderiam estar relacionados com a espessa camada de cera observada na superfície foliar desta planta daninha (Atkinson, 2014).

Martins et al. (2012) não observaram controle de $C$. benghalensis com a utilização de glyphosate ( 1.080 g e.a. ha $\left.{ }^{-1}\right)$, seja isolado ou em associação com imazethapyr. Em trabalho realizado por Procópio et al. (2007) foi observado que a aplicação isolada de glyphosate (960 g e.a. ha ${ }^{-1}$ ) apresentou baixo nível de controle para $C$. benghalensis (40\%) aos 25 DAA, mas quando foi adicionado imazethapyr $\left(50 \mathrm{~g}\right.$ i.a. ha $\left.{ }^{-1}\right)$ o controle aumentou para $60 \%$, porém sendo ainda considerado um controle não eficiente. Para controle de Commelina cummins, somente em aplicações com doses superiores a 3.000 g e.a. ha ${ }^{-1}$ de glyphosate foram eficientes (Ulloa e Owen, 2009).

Esses resultados apontam para a necessidade de revisão por parte dos órgãos governamentais do registro de uso de glyphosate para controle de $M$. nudiflora, pois os resultados demonstram controle muito abaixo de $80 \%$, requisito mínimo para se obter o registro de uso no Brasil (MAPA, 2016b).

Em vista dos elevados níveis de controle de $M$. nudiflora decorrentes da aplicação de atrazine, presume-se que as características de crescimento por estolões tenham propiciado maior absorção deste herbicida, uma vez que, possa ter ocorrido via absorção radicular. Como descrito por Maciel et al. (2002) plantas de Brachiaria plantaginea foram susceptíveis a atrazine também a partir de absorção radicular, com a presença de umidade no solo, ocasionando controle tão eficiente quanto os tratamentos que receberem aplicação foliar. $\mathrm{O}$ mesmo ocorreu para plantas de $M$. nudiflora em que Yu e McCullought (2016) relataram que a absorção do herbicida via radicular tem fundamental significância para o aumento de controle com atrazine, sendo importante via de entrada para o herbicida na planta, visto que a absorção foliar é limitada pelas características da cutícula da folha, que reduzem a absorção do herbicida (Monquero et al., 2004).

\section{Experimento 2. Dose resposta de atrazine para controle de $M$. nudiflora}

Não foram observadas diferenças significativas entre os tratamentos para as variáveis-resposta porcentagem de cobertura do solo e massa seca de $M$. nudiflora antes da aplicação dos tratamentos herbicidas, evidenciando a uniformidade da área experimental em relação à distribuição das plantas daninhas (Tabela 3).

Tabela 3. Resumo da análise de variância para porcentagem de cobertura e massa seca de $M$. nudiflora. Experimento 2. Nova Xavantina (MT), 2010.

\begin{tabular}{lccc}
\hline \multicolumn{1}{c}{ Variáveis } & QM $_{\text {TRAT }}$ & CV (\%) & Média Geral \\
\hline Porcentagem de cobertura de $M$. nudiflora & $46,25^{\mathrm{ns}}$ & 16,99 & 76,25 \\
Massa seca de $M$. nudiflora & $16049,93^{\mathrm{ns}}$ & 32,98 & 591,66 \\
\hline ns Não significativo a 5\% de probabilidade pelo teste F & &
\end{tabular}

Na Figura 2, é apresentada a regressão linear para controle de $M$. nudiflora em função das doses de atrazine. Observa-se que aos 7 DAA, o tratamento com a maior dose utilizada (1.540 g i.a. ha ${ }^{-1}$ de atrazine) apresentou o maior índice de controle com aproximadamente $60 \%$. Aos 14 DAA apenas as doses de 1.320 e 1.540 $\mathrm{g}$ i.a. ha ${ }^{-1}$ de atrazine apresentaram controle eficiente (> 80\%), porém na última avaliação aos 28 DAA, todos os tratamentos apresentaram eficiência superior a 96\%. Dessa forma, para propiciar controle eficiente aos 28 DAA, a menor dose avaliada de atrazine (880 g i.a. ha ${ }^{-1}$ ) é suficiente para o controle de $M$. nudiflora.

Avaliando o controle de $M$. nudiflora $\mathrm{Yu}$ e McCullough (2016), constataram eficiência pela atrazine, onde doses de 1.120 g i.a. ha ${ }^{-1}$ promoveram níveis de controle de 95\%, ao passo que no presente trabalho, estes níveis foram observados na dose de 880 g i.a. ha ${ }^{-1}$. 


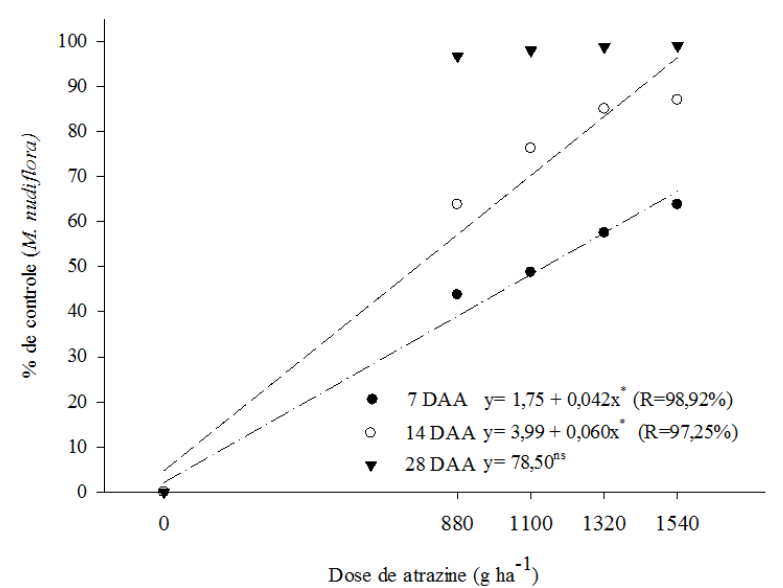

Figura 2. Regressão linear para controle de Murdannia nudiflora, nos tempos de avaliação de 7, 14 e 28 DAA, em função de doses crescentes de atrazine. Experimento 2. Nova Xavantina (MT), 2010.

O uso de atrazine é difundido no sistema agrícola brasileiro. $\mathrm{Na}$ cultura do milho para manejo de plantas daninhas em pós-emergência, se utiliza principalmente a atrazine, que além de controlar a soja voluntária, auxilia no controle de outras plantas daninhas como corda-de-viola (Commelina spp.) e outras folhas largas (Duarte et al., 2007). Outras estratégias de manejo como adoção de culturas de cobertura e rotação de herbicidas com mecanismos de ação distintos devem ser adotadas para o controle de $M$. nudiflora, a fim de preservar a eficiência da atrazine e diversificar as opções de manejo para esta planta daninha em outras culturas.

\section{Conclusões}

Glyphosate não apresenta controle eficiente de $M$. nudiflora quando aplicado isolado na dose de $972 \mathrm{~g}$ e.a. ha ${ }^{-1}$, ou em mistura com imazethapyr (30 g i.a. ha $\left.{ }^{-1}\right)$. A aplicação isolada de imazethapyr (100 g i.a. ha $\left.{ }^{-1}\right)$ ou carfentrazone (12 g i.a. ha ${ }^{-1}$ ), não controla $M$. nudiflora, apresentando controle eficiente apenas quando associado a atrazine.

O herbicida atrazine isolado a partir da dose de 880 g i.a. ha ${ }^{-1}$ é eficiente para o controle de $M$. nudiflora.

\section{Referências}

Ahmed, S.; Opeña, J.L.; Chauhan, B.S. Seed germination ecology of doveweed (Murdannia nudiflora) and its implication for management in dry-seeded rice. Weed Science, v.63, n.2, p.491-501, 2015.

Atkinson J. Biology, Ecology, and Control of Doveweed (Murdannia nudiflora [L.] Brenan). 2014. 115 f. PhD Dissertation - Clemson University, SC, 2014.

Braz, G.B.P.; Constantin, J.; Oliveira Jr, R.S.; Oliveira Neto, A.M., Dan, H.A.; Guerra, N.; Jumes, T.M.C. Desempenho de herbicidas utilizados no algodoeiro para o controle de Amaranthus. Revista Brasileira de

Herbicidas, v.11, n.1, p.1-10, 2012.

Chauhan, B.S.; Abugho, S.B. Effect of crop residue on seedling emergence and growth of selected weed species in a sprinkler-irrigated zero-till dry-seeded rice system. Weed Science, v.61, n.3, p.403-409, 2013.

Cruz, C.D. Genes Software - extended and integrated with the R, Matlab and Selegen. Acta Scientiarum, v.38, n.4, p.547-552, 2016.

Cruz, D.L.S.; Rodrigues, G.S.; Dias, F.O., Alves, J.M.A.; Albuquerque, J.D.A.A. Levantamento de plantas daninhas em área rotacionada com as culturas da soja, milho e arroz irrigado no cerrado de Roraima. Revista Agro@mbiente On-line, v.3, n.1, p.58-63, 2009.

Cury, J.P.; Santos, J.B.; Silva, E.B.; Byrro, E.C.M.; Braga, R.R.; Carvalho, F.P.; Valadao Silva, D. Acúmulo e partição de nutrientes de cultivares de milho em competição com plantas daninhas. Planta Daninha, v.30, n.2, p.287296, 2012.

Dias, A.C.R.; Carvalho, S.J.P.; Christoffoleti, P.J. Fenologia da trapoeraba como indicador para tolerância ao herbicida glyphosate. Planta Daninha, v.31, n.1, p.185-191, 2013.

Duarte, A.P.; Silva, A.C.; Deuber, R. Plantas infestantes em lavouras de milho safrinha, sob 
diferentes manejos, no médio Paranapanema. Planta Daninha, v.25, n.2, p.285-291, 2007.

Erasmo, E.A.L.; Terra, M.A.; Costa, N.V.; Domingos, V.D.; Didonet, J. Fenologia e acúmulo de matéria seca em plantas de Murdannia nudiflora durante seu ciclo de vida. Planta Daninha, v.21, n.3, p.397-402, 2003.

Erasmo, E.A.L.; Terra, M.A.; Domingos, V.D.; Martins, C.C.; Costa, N.V. 2008. Superação da dormência em sementes de Murdannia nudiflora (L.) Brenan. Acta Scientiarum Agronomy, v.30, n.2, p.273-277, 2008.

Ferraresi, D.A.; Yamashita, O.M.; Carvalho, M.A.C. Superação da dormência e qualidade de luz na germinação de sementes de Murdannia nudiflora (L.) Brenan. Revista Brasileira de Sementes, v.31, n.4, p.126-132, 2009.

Kissmann, K.G.; Groth, D. Plantas infestantes e nocivas. São Paulo: BASF, 1997, Tomo 1, ed.2, p.110.

Lorenzi, H. Commelinaceae. (Ed. 4). Plantas daninhas do Brasil: terrestres, aquáticas, parasitas e tóxicas. Nova Odessa: Editora Plantarum, 2008. p.230-236.

Luz, F.N.; Yamashita, O.M.; Ferraresi, D.A.; Carvalho, M.A.C.; Campos, O.R.; Koga, P.S.; Massaroto, J.A. Interferência de luz, temperatura, profundidade de semeadura e palhada na germinação e emergência de Murdannia nudiflora. Comunicata Scientiae, v.5, n.1, p.26-33, 2014.

Maciel, C.D.G.; Constantin, J.; Oliveira Jr, R.S.; Farias, A. Método alternativo de avaliação da absorção de atrazine por plantas de Brachiaria plantaginea. Planta Daninha, v.20, n.3, p.431438, 2002.

MAPA, Ministério da Agricultura, Pecuária e Abastecimento. Agrofit. Disponível em:< http://agrofit.agricultura.gov.br/agrofit_cons/pr incipal_agrofit_cons >>. Acesso em: jul. 2016a.

MAPA, Ministério da Agricultura, Pecuária e Abastecimento. Manual de procedimentos de registro de agrotóxicos. Disponível em:< http://www.agricultura.gov.br/arq_editor/file/v egetal/agrotoxicos/Manual\%20de\%20Procedi mentos\%20para\%20Registro\%20de\%20Agrot \%C3\%B3xicos.pdf $>>$. Acesso em: nov. 2016b.

Martins, D.; Santana, D.C.; Souza, G.S.F.; Bagatta, M.V.B. Manejo químico de espécies de trapoeraba com aplicação isolada e em mistura de diferentes herbicidas. Revista Caatinga, v. 25, n.2, p. 21-28, 2012.

Monquero, P.A.; Christoffoleti, P.J.; Osuna, M.D.; Prado, R.A. Absorption, translocation and metabolism of glyphosate by plants tolerant and susceptible to this herbicide. Planta Daninha, v.22, n.3, p.445-451, 2004.

Norsworthy, J.K.; Ward, S.M; Shaw, D.R.; Lewellyn, R.S.; Nichols, R.L.; Webster, T.M.; et al. Reducing the Risks of Herbicide Resistance: Best Management Practices and Recommendations. Weed Science, v.60, sp.1, p.31-62, 2012.

Osipe, J.B.; Silva Teixeira, E.; Osipe, R.; Fonseca Sorace, M.A.; Cossa, C.A.; Oliveira Neto, A.M. Associação de sulfentrazone e glyphosate para o controle de plantas daninhas na cultura da soja $\mathrm{RR}^{\circledR}$. Revista Brasileira de Herbicidas, v.7, n.1, p.15-25, 2008.

Procópio, S.O.; Menezes, C.C.E.; Betta, L.; Betta, M. Utilização de chlorimuron-ethyl e imazethapyr na cultura da soja Roundup Ready ${ }^{\circledR}$. Planta Daninha, v.25, n.2, p.365-373, 2007.

SOCIEDADE BRASILEIRA DA CIÊNCIA DAS PLANTAS DANINHAS - SBCPD. Procedimentos para instalação, avaliação e análise de experimentos com herbicidas. Londrina: 1995.42 p.

Ulloa, S.M.; Owen, M.D. Response of Asiatic dayflower (Commelina communis) to glyphosate and alternatives in soybean. Weed Science, v.57, n.1, p.74-80, 2009.

Wilson Jr., D.G.; Burton, M.G.; Spears, J.F.; York, A.C. Doveweed (Murdannia nudiflora) germination and emergence as affected by 
temperature and seed burial depth. Weed science, v.54, n.6, p.1000-1003, 2006.

$\mathrm{Yu}$, J.; McCullought, P.E. Efficacy and Fate of Atrazine and Simazine in Doveweed (Murdannia nudiflora). Weed Science, v.64, n.3, p.379-388, 2016. 